\title{
Security Analysis of Image-Based PUFs for Anti-counterfeiting
}

\author{
Saloomeh Shariati, François Koeune, and François-Xavier Standaert \\ ICTEAM Institute, Electrical Engineering Department \\ Université Catholique de Louvain, Place du Levant, 3, \\ B-1348 Louvain-la-Neuve, Belgium \\ \{saloomeh.shariati,francois.koeune,fstandae\}@uclouvain.be
}

\begin{abstract}
Physically Unclonable Functions are a promising tool to protect against counterfeiting attacks. Yet, as with any security system, it is important to embed them in a sound protocol, ensuring that no unexpected weakness is present in the "mortar" binding the components together. This paper proposes an anti-counterfeiting protocol that provably reduces to natural properties of its underlying components, namely an image-based Physical Function System bearing physical unclonability and an existentially unforgeable signature scheme. Experiments confirm the practical feasibility of our construction.
\end{abstract}

\section{Introduction}

Counterfeiting of trademarked products is a rapidly growing problem for the worldwide economy. Two types of threats are to be faced. On the one hand, Insider Counterfeiting (manufacturer overproduction) refers to unauthorized production by the legitimate manufacturer who realizes profits by producing extra quantities outside their license agreement [1]. On the other hand, Outsider Counterfeiting refers to unauthorized reproduction of products by other counterfeiters. Many ad-hoc methods have been proposed to avoid counterfeiting. Examples include so-called overt physical identifiers such as hologram and inks that visibly alter under light, or so-called covert technology such as invisible inks, proprietary photonic inks [2] and Radio Frequency Identification (RFID) Tags [3].

Recently [4, 5], Physically Unclonable Functions, or PUFs, have been introduced and, among many other applications, proposed as an effective tool for anti-counterfeiting systems. A Physically Unclonable Function (PUF) is a function that is embodied in a physical structure and is easy to evaluate, but hard to clone. Generally, a PUF interacts with stimuli (challenges) in an intricate way, and leads to unique and unpredictable responses. As a particular example, image-based PUFs are based on random visual features assessed by an imaging method. These can be employed for identification purposes [6].

For anti-counterfeiting applications, the core concept of using PUF primitives is to rely on the unique physical properties to identify a product. The PUF can be either intrinsic in the product or extrinsic and glued to the product. The general idea is to digitally sign the product information (e.g. EPC code) together

B. De Decker and D.W. Chadwick (Eds.): CMS 2012, LNCS 7394, pp. 26-38, 2012.

(C) IFIP International Federation for Information Processing 2012 
with the information extracted from the embedded PUF and use this signature as the certificate of authenticity [7-11].

Various image-based PUFs have been proposed to be applied in anti-counterfeiting systems, in different contexts and under different assumptions [10, 12 16. In this paper, we propose a unified formal treatment of the use of image-based PUFs as a counterfeiting prevention tool. Starting with a description of an image-based Physical Function System, we define a secure anti-counterfeiting scheme and provide a construction meeting this definition. The construction combines physical protection blocks with cryptographic protection blocks. We define an attack model and derive the security property a PUF must fulfill in order to be eligible as a secure physical building block. We prove that this security property is equivalent to the physical unclonability property that was defined in [17]. We finally illustrate our model by studying a practical example.

The rest of the paper is structured as follows: Section 2 briefly describes previous works. In Section 3, we bring the previous approaches to the formalization of a Physical Function System and particularly image-based Physical Function System. In Section 4, we present an informal view of the anti-counterfeiting scheme and the security assumptions which is followed by the formal definition of anti-counterfeiting scheme in Section 5. Then we provide the attack model and formalization of the security of the anti-counterfeiting scheme in Section 6 Section 7 discusses application on a practical example.

\section{Previous Work}

Early works that exploit the physical properties of random structures for authentication purposes date back to [18, 19. The term Physically Unclonable Function was introduced by Pappu [4,5]. Since then, many different physical objects have been proposed as PUF candidates, including Optical PUF [4, 5, Coating PUF [20, Silicon PUF [21 23], SRAM PUF [24], Paper PUF 10, 12 14], Phosphor PUF [3, 15, Laser-Written PUF [16, 25, etc.

Various application fields have also been proposed, such as secure key generation [26, 27], key storage [28], or in the design of block ciphers [29]. The idea of combining cryptographic means such as digital signature together with information extracted from the embedded PUF for authentication purpose was first applied in [7-11,26].

\section{Background}

Armknecht et al. proposed a generic security framework of physical functions [17. They explored the physical functions in general, where unclonability is only one possible security property. We briefly describe the components of the framework which will be used in anti-counterfeiting scheme afterwards. For a detailed description of each component we refer to [17]. A Physical Function $(P F)$ consists of a physical component $p$ and an evaluation procedure Eval. A 
$\mathrm{PF}\left(\mathrm{PF}_{p, \alpha_{\mathrm{PF}}}: \mathcal{X} \rightarrow \mathcal{Y}\right)$ takes as input a challenge $x$ and outputs a response $y$. The challenge-response behavior of a PF relies on the properties of the physical component $p$, an evaluation parameter $\alpha_{\mathrm{PF}}$ and some evaluation noise (measurement uncertainties). A Physical Function is a probabilistic procedure because on a single challenge, it may produce different responses due to the evaluation noise. Since the output of the PF is noisy, usually it is combined with an extraction algorithm Extract with an extraction parameter $\alpha_{\mathrm{EX}}$ that compensates a certain amount of noise and provides robust output. In addition to the response $y$, Extract also takes as input some helper data $h$ generated the first time $p$ was evaluated (i.e. in setup mode) and helping noise removal. The combination of $\mathrm{PF}$ and extraction algorithm is considered as one single building block which is defined as:

Definition 1 (Physical Function System [17]). A physical function system PFS is a probabilistic procedure

$$
\mathrm{PFS}_{p, \alpha_{\mathrm{PF}}, \alpha_{\mathrm{EX}}}: \mathcal{X} \times(\mathcal{H} \cup\{\epsilon\}) \rightarrow \mathcal{Z} \times \mathcal{H}
$$

where $\mathcal{X}$ is the set of challenges, $\mathcal{H}$ the set of helper data values, $\epsilon$ the empty string, and $\mathcal{Z}$ the set of outputs. Internally, a PF system is the combination of a physical function and an extraction algorithm Extract, i.e.,

$$
\begin{aligned}
& \operatorname{PFS}_{p, \alpha_{\mathrm{PF}}, \alpha_{\mathrm{EX}}}(x, h) \\
& =\operatorname{Extract}_{\alpha_{\mathrm{EX}}}\left(\operatorname{PF}_{p, \alpha_{\mathrm{PF}}}(x), h\right) \rightarrow\left(z, h^{\prime}\right)
\end{aligned}
$$

If $h \neq \epsilon$, then $h^{\prime}=h$. Only in case $h=\epsilon$, a new helper data $h^{\prime}$ is generated for $x$. In the following, we omit the internal components and abbreviate PFS = $\mathrm{PFS}_{p, \alpha_{\mathrm{PF}}, \alpha_{\mathrm{EX}}}$.

Note that $h=\epsilon$ means that Extract should be executed in setup mode to generate a new helper data $h$ w.r.t. challenge $x$. In case $h \neq \epsilon$, Extract should be executed in reconstruction mode to recreate output $z$ associated with challenge $x$ and helper data $h$.

In [6], the authors specialize the general Physical Function System for the specific case of image-based PUFs. The reason for specialization in image-based PUFs is twofold. First, the application of image-based PUFs is specific and is mainly for anti-counterfeiting systems. As a matter of fact, unlike some PUFs (e.g., optical PUFs), the input to image-based PUF is usually a fixed challenge and therefore a mathematical clone (a mathematical procedure that yields the same challenge-response behavior as the PUF e.g., a fake image) can be created by imitating the response of the PUF to this challenge (this is further discussed in Section 4.1). Secondly, the response of the image-based PUF is a real-valued image and require that a specific processing (i.e., dimensionality reduction and binarization) be integrated with the extraction procedure. Fig. 1 illustrates image-based PF system in setup and reconstruction mode.

An image-based PF System includes an image-based PF with a fixed challenge $(\mathcal{X}=x)$ and an image-based Extraction. In set-up mode (Fig. 1(a)), image 


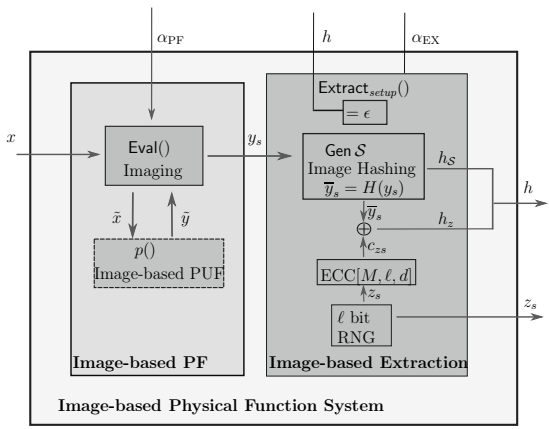

(a) Setup mode.

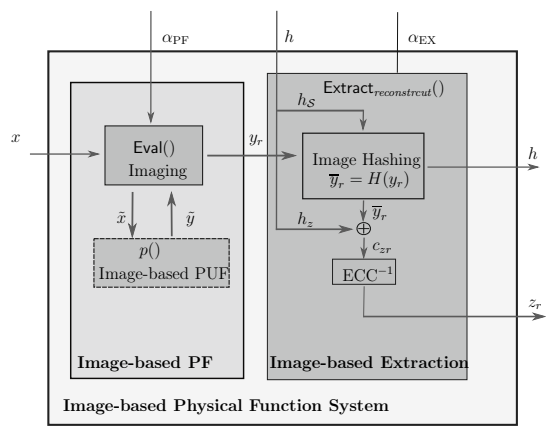

(b) Reconstruction mode.

Fig. 1. Image-based Physical Function system [6]

hashing compresses and binarizes the response of the image-based PF $y_{s}$ and produces the image hash $\bar{y}_{s}$. Then a typical fuzzy extraction called Code-Offset fuzzy extraction [30 is applied. It masks the image hash $\bar{y}_{s}$ with a random codeword $C_{z s}$ of a predefined Error Correcting Code (ECC) and generates output $z_{s}$ and helper data $h$. In reconstruction mode, the response of the PF to the same challenge is evaluated $y_{r}$ and image hashing generates image hash $\bar{y}_{r}$. Then ouput $z_{r}$ is reconstructed using the image hash $\bar{y}_{r}$, second part of helper data $h_{z}$ and ECC decoding as depicted in Fig. 1(b). For a more in depth view of the components of an image-based Physical Function System, we refer to [6].

\section{Informal Description of Anti-counterfeiting Scheme}

In this section, a general view of the components of the anti-counterfeiting scheme and the security assumptions are described. The components of the scheme will be discussed in detail in the following section.

1. A configuration step Config is performed by the trademark owner and the PUF provider. They decide about the type of PUF, the parameters of the system, etc. A private/public key pair is also established in this step.

2. The PUF provider runs a creation process Create and delivers the created PUFs to the trademark owner.

3. A registration step Reg is performed by the trademark owner. It includes extracting digital information from the PUF and digitally signing this information plus some side information (e.g. serial number) about the product. Depending on the context, this step can take place either after the product to protect has been manufactured, or before: the PUF and registration data can for example be shipped to the manufacturer to be later physically or logically bound to the product. The PUF and data could for example be glued and printed on the product, or on a certificate of authenticity accompanying it. The product and PUF are then delivered to the market. 
4. A verification process Verif is performed each time someone wants to determine whether the embedded PUF of a given product is authentic or not. Examples of entities performing the verification process includes: trademark owner, customs, wholesaler or retailer and end-user.

\subsection{Security Assumptions}

1. As a classical property of physically unclonable functions, we expect that, due to uncontrollable variations during the manufacturing process, PUFs cannot be physically cloned, i.e. that it is practically impossible/very costly for an adversary to generate pairs of PUFs yielding identical behavior when queried. We will provide an explicit phrasing of this expectation later in this paper (Eq. 8), and describe a testing methodology in Section 7.2. A PUF is usually employed to protect products whose value is less than the cost of cloning the PUF. As technology grows, we may expect that the cost of cloning a PUF does not anymore exceed the product value. As a consequence, the PUF-based system may need upgrade by time.

2. Image-based PUFs are physically unclonable and not necessarily mathematically unclonable. Mathematical unclonability means that it should be very hard to construct a mathematical procedure that yields the same challengeresponse behavior as that of the PUF. Image-based PUFs in general do not have this property and a mathematical clone (e.g. a fake image) can be created by imitating the response (image) of the PUF. As a consequence, we assume that any verification process involves a prior verification that a real PUF, and not a mathematical clone (e.g. a picture) is being dealt with.

3. The various steps of the anti-counterfeiting scheme, i.e. Config, Create, Reg and Verif are performed by trusted parties, using trusted parameters. In particular, this implies that registration is only performed on physical components originating from a trusted source.

4. We authenticate the PUF and not the product. This is equivalent to authenticating the product itself when the PUF is inherently part of the product (intrinsic PUF). It is not necessarily the case when the PUF is a distinct object attached to the product (extrinsic PUF), but is still be sufficient in most anti-counterfeiting scenarios (both insider and outsider), where the trademark owner mostly wants to control the amount of products delivered to the market1.

The above assumptions lay down a sound framework for implementing a secure PUF-based system. Let us briefly discuss some of their consequences.

As usual, we assume (assumption 1) that the PFS system in use generates inherently unique tags. However, it is difficult to ensure that, by modifying the generation parameters, an adversary will not be able to come up with a degraded

${ }^{1}$ For other scenarios, where an inseparable link between the PUF and the product is necessary, intrinsic PUFs, or additional measures such as using a tamper-proof seal would be necessary. 
version of the PFS that will trigger collisions2. This could yield collision-based attacks, in a way very similar to hash-function collision attacks against signature schemes, where an adversary generates degraded tags, gets one of them registered, and can use this as a registration proof of the other ones. Checking whether a given tag was produced using the appropriate parameters is not always obvious for the registration authority or verifier. Assumption 3 allows us to get basically rid of that concern, as we assume that the only tags that will be registered are those produced by a trusted source, and thus implicitly using the correct parameters. We are thus left with the much more natural assumption that tags produced using different parameters would be sufficiently different to be sure that they cannot induce a collision with the "honest" ones. Of course, the validity of this "natural" assumption should still be asserted by the system designer when selecting a specific PUF realization.

It is worth noting that, although we assume above that the verification is performed by a trusted party, this trust is in fact only limited. An untrusted verifier is impossible to capture in a security model, in the sense that it is impossible to prevent a rogue verifier from simply providing a positive answer to any verification request. Nevertheless, the use of asymmetric cryptography allows storing only public, non-critical keys on the verifier's side. So a compromised verifier can (inevitably) be used to provide incorrect information to its user, but it cannot be exploited to affect the system's global security.

\section{Formal Model of Anti-counterfeiting Scheme}

Let us now formally define our anti-counterfeiting scheme. In addition to the aforementioned PFS, it relies on a signature scheme providing existential unforgeability under an adaptive chosen messages attack. Intuitively, this means that it is impossible for an adversary running in "reasonable" time to produce the signature of a new message, even if he can beforehand request the signature of many messages that he chooses. We refer to [31] for a complete definition of this security property, which is a classical requirement of signature schemes.

Definition 2. The anti-counterfeiting scheme $\Pi$ includes tuple of four processes (Config, Create, Reg, Verif) satisfying the following:

1. Config: The configuration process is performed one time and determines the type of PUF and the parameters of the system including the fixed challenge $x$, creation parameter $\alpha_{\mathrm{CR}}$, evaluation parameter $\alpha_{\mathrm{PF}}$, extraction parameter $\alpha_{\mathrm{EX}}$ and a pair of private key $s k$ and public key $p k$.

2. Create $\left(\alpha_{\mathrm{CR}}\right) \rightarrow p$. The creation process takes as input the creation parameter $\alpha_{\mathrm{CR}}$ and outputs the physical component $p$ based on the creation parameter $\alpha_{\mathrm{CR}}$.

${ }^{2}$ As an extreme example, consider modifying an image-based PUF so that all produced tags are uniformly black. 
3. $\operatorname{Reg}_{x, \alpha_{\mathrm{PF}}, \alpha_{\mathrm{EX}}, s k}(p, A u x) \rightarrow(\sigma, h, A u x)$. The registration process $\operatorname{Reg}_{x, \alpha_{\mathrm{PF}}, \alpha_{\mathrm{EX}}, s k}$ takes as input the physical component $p$ and optional auxiliary data. Auxiliary data $A u x$ includes side information about the PUF such as its serial number or EPC, creation date, expiration date, distribution points, etc. Since the challenge $x, \alpha_{\mathrm{PF}}, \alpha_{\mathrm{EX}}$ and the private key $s k$ are fixed and implicitly trusted, we usually discard them in our notation, that is we simply write $\operatorname{Reg}$ instead of $\operatorname{Reg}_{x, \alpha_{\mathrm{PF}}, \alpha_{\mathrm{EX}}, s k}$. The registration process Reg is performed in two phases:

1) The set-up mode of the image-based PF system (See Fig. 1(a) is executed for $p$ using $x, \alpha_{\mathrm{PF}}$ and $\alpha_{\mathrm{EX}}$, yielding the output $z$ and helper data $h$ as:

$$
\operatorname{PFS}(x, \epsilon) \rightarrow(z, h)
$$

2) The output, helper data and auxiliary data are signed using the private key $s k$, yielding the signature $\sigma=\operatorname{Sign}_{s k}(z\|h\| A u x)$.

4. Verif ${ }_{x, \alpha_{\mathrm{PF}}, \alpha_{\mathrm{EX}}, p k}(p, h, A u x, \sigma) \rightarrow b \in\{0,1\}$. The verification process Verif $_{x, \alpha_{\mathrm{PF}}, \alpha_{\mathrm{EX}}, p k}$ takes as input a physical component $p$, helper data $h$, auxiliary data $A u x$ and signature $\sigma$, and outputs a validity bit $b$. Hereafter, we simply write Verif instead of Verif $x, \alpha_{\mathrm{PF}}, \alpha_{\mathrm{Ex}}, p k$. The verification process Verif is also performed in two phases:

1) The reconstruction mode of image-based PF system (See Fig. 1(b)] is executed for $p$ using $x, \alpha_{\mathrm{PF}}, \alpha_{\mathrm{EX}}$ and the helper data $h$. The output $z_{r}$ is generated as following:

$$
\operatorname{PFS}(x, h) \rightarrow\left(z_{r}, h\right)
$$

2) The verification algorithm of the signature scheme is executed on $\left(z_{r}\|h\| A u x\right)$ using the public key $p k$ to check the authenticity of the PUF. The verification algorithm of the signature scheme outputs a bit $b$, with $b=1$ meaning signature valid and $b=0$ meaning signature invalid.

The verification process outputs the bit $b$.

\section{Attack Model and Security}

For our security analysis, we assume that the Adversary A has access to an oracle CReg(.). When it is queried with parameter $\alpha_{\mathrm{CR}, \mathrm{i}}$, this oracle checks whether $\alpha_{\mathrm{CR}, \mathrm{i}} \in \mathcal{A}_{\mathrm{CR}}$ and, if it does, creates $p_{i}$, registers the created $p_{i}$ and returns $\left(p_{i}, \sigma_{i}, h_{i}, A u x_{i}\right)$ (Fig. 2).

In practice, only adversaries with bounded time and computational effort are relevant. Thus we consider only PPT adversaries and additionally limit them to query the CReg oracle at most $q$ times.

Definition 3 (Attack Model). Let $\Pi=$ (Config, Create, Reg, Verif) be the anti-counterfeiting scheme. Adversary A is given pk and oracle access to CReg(.) as defined above. Let $Q$ be the set of pairs ( $p$, Aux) that were generated by 
Adversary A

Oracle Creg(.)

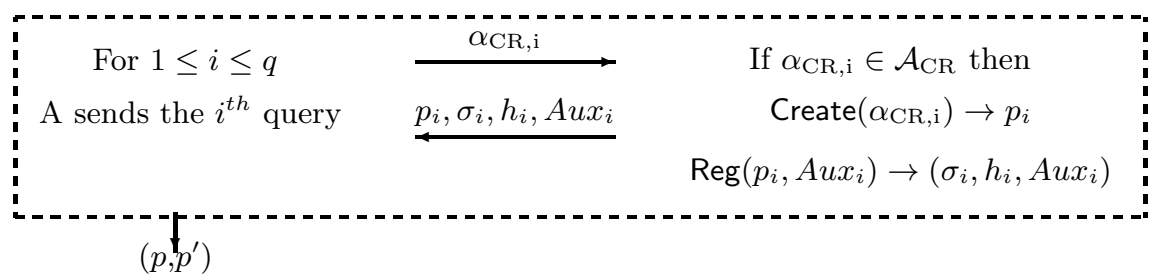

Fig. 2. Counterfeiting experiment Counterfeit ${ }_{\mathrm{A}, \Pi}(q)$

CReg(.). The adversary A then generates a set of $\left(p^{\prime}, h^{\prime}, A u x^{\prime}, \sigma^{\prime}\right)$. The output of the counterfeiting experiment Counterfeit $\mathrm{A}, \Pi(q)$ is defined to be 1 if

$$
\operatorname{Verif}\left(p^{\prime}, h^{\prime}, A u x^{\prime}, \sigma^{\prime}\right) \rightarrow 1 \quad \text { and } \quad\left(p^{\prime}, A u x^{\prime}\right) \notin Q \text {. }
$$

The security of the anti-counterfeiting scheme is then defined as follows:

Definition 4 (Security). The anti-counterfeiting scheme $\Pi$ is $\beta$-secure if for all probabilistic polynomial-time (PPT) adversaries, we have:

$$
\operatorname{Pr}\left[\text { Counterfeit }_{\mathrm{A}, \Pi}(q)=1\right] \leq \beta
$$

Lemma 1. Suppose that the signature scheme is existentially unforgeable under an adaptive chosen message attack. If the output of the adversary experiment is 1 , then there exists at least one instance $p_{i}$ such that there has been a registration step:

$$
\operatorname{Reg}\left(p_{i}, A u x_{i}\right) \rightarrow\left(\sigma_{i}, h_{i}, A u x_{i}\right)
$$

and $\sigma^{\prime}=\sigma_{i}, h^{\prime}=h_{i}, A u x^{\prime}=A u x_{i}$ and $z^{\prime}=z_{i}$ s.t $\operatorname{PFS}\left(x, h_{i}\right) \rightarrow\left(z^{\prime}, h_{i}\right)$.

Proof. Suppose that there does not exist any $p_{i}$ that has been registered by a trusted authority such that $\sigma^{\prime}=\sigma_{i}, h^{\prime}=h_{i}, A u x^{\prime}=A u x_{i}$ and $z^{\prime}=z_{i}$. Then, $\operatorname{Verif}\left(p^{\prime}, h^{\prime}, A u x^{\prime}, \sigma^{\prime}, p k\right) \rightarrow 1$ implies that the adversary succeed in producing a valid signature pair $\left(\left(z^{\prime}\left\|h^{\prime}\right\| A u x^{\prime}\right), \sigma^{\prime}\right)$ for a signature that has not been produced by the signature scheme. This contradicts the existential unforgeability assumption for the signature scheme.

As a direct consequence of Lemma 1 the only way for the adversary to make a counterfeit is to produce a physical component $p^{\prime}$ that collides with a previously registered physical component $p_{i}$, i.e. provides the same output using its corresponding helper data $h_{i}$. Hence, the security property of the anti-counterfeiting scheme can be evaluated as follows:

Property 1. The anti-counterfeiting scheme $\Pi$ is $\beta$-secure (w.r.t. $x$ ) if for all PPT adversaries $\mathrm{A}$ that are limited to calling the CReg oracle at most $q$ times, it holds that: 


$$
\begin{array}{r}
\operatorname{Pr}\left[\operatorname{PFS}^{\prime}(x, h) \rightarrow(z, h): \operatorname{PFS}(x, \epsilon) \rightarrow(z, h)\right. \\
\operatorname{Counterfeit}_{\mathrm{A}, \Pi}(q) \rightarrow\left(p, p^{\prime}\right) ; \\
\left.p \in\left[\text { Create }\left(\alpha_{\mathrm{CR}} \in \mathcal{A}_{\mathrm{CR}}\right)\right] ; p^{\prime} \in\left[\operatorname{Create}\left(\alpha_{\mathrm{CR}}^{\prime} \in \mathcal{A}_{\mathrm{CR}}\right)\right]\right] \leq \beta
\end{array}
$$

where ':' denotes the conditional probability and PFS' is the PF system using $p^{\prime}$. The security experiment Counterfeit $\operatorname{A}, \Pi_{(}(q)$ is depicted in Fig. 2

This corresponds to the existential physical unclonability property that was defined in [17.

\section{Implementation}

Let us illustrate this on an example. For this purpose, we first need to briefly discuss another property, namely, the construction's robustness.

\subsection{Robustness}

So far, we emphasized on the security of the anti-counterfeiting system. However, for the system to be useful in practice, we should also ensure that outputs are reproducible, i.e. that different evaluations of a single PUF produce the same output. Robustness [17] expresses the probability that the output generated by the reconstruction phase matches the value generated in the set-up phase and is formally defined as:

Definition 5 (Robustness). Let PFS be a PF system (Definition 1) and let $x \in \mathcal{X}$ be a challenge. The robustness of PFS (w.r.t. $x$ ) is defined as the probability

$$
\begin{array}{r}
\rho_{\mathrm{PFS}}(x):=\operatorname{Pr}[\operatorname{PFS}(x, h) \rightarrow(z, h): \\
\operatorname{PFS}(x, \epsilon) \rightarrow(z, h)]
\end{array}
$$

A sound evaluation of a practical anti-counterfeiting system requires assessing concurrently its security and its robustness.

\subsection{Testing Method}

As discussed in Section 4, the assumption that only tags produced by a trusted manufacturer are registered can often simplify unclonability testing. Relying on the assumption that different creation parameters will not trigger collisions with tags produced using $\alpha_{\mathrm{CR}}$, we can rewrite Eq. 8 as:

$$
\begin{array}{r}
\operatorname{Pr}\left[\operatorname{PFS}^{\prime}(x, h) \rightarrow(z, h): \operatorname{PFS}(x, \epsilon) \rightarrow(z, h)\right. \\
\left(p, p^{\prime}\right) \in\left[\operatorname{Create}\left(\alpha_{\mathrm{CR}} \in \mathcal{A}_{\mathrm{CR}}\right)\right] \leq \beta ;
\end{array}
$$


and thus focus on the probability that an honest manufacturer creates two clones by coincidence.

Following the method of [6], this probability can be statistically estimated by sampling $R$ PUFs and their corresponding pictures. First, one PUF is chosen as a "target", the image of which is used in set-up mode to generate helper data $h$ and output $z_{s}$. The remaining $(R-1)$ images, together with the initial helper data $h$, are then used in reconstruction mode, and the generated outputs are compared with $z_{s}$. This experiment is repeated $R$ times, each PUF being selected once as target. The security property $\beta$ is then estimated by:

$$
\operatorname{Pr}\left[z_{r}=z_{s}: p \neq p^{\prime}\right] \leq \beta
$$

Similarly, robustness can be evaluated from Eq. 9, using a dataset of PUFs that are evaluated several times with different observation noise. Given a dataset of $P$ different PUFs observed $Q$ times each, the robustness can be statistically estimated as follows. For each PUF, one observation of the PUF is used in set-up mode to produce output $z_{s}$ and helper $h$. The remaining $(Q-1)$ observations, together with the initial helper data $h$, are then used in reconstruction mode, and the generated outputs are compared with $z_{s}$. This is repeated $Q$ times, with each observation being once in the set-up phase. The robustness is then estimated from $\operatorname{Pr}\left[z_{r}=z_{s}\right]$ that is equivalent to Eq. 9.

\subsection{Practical Example}

As a practical example, we implemented an image-based Physical Function System based on a Laser-Written PUF (LPUF). The basic principle consists in engraving tiny laser marks on the surface or volume of a transparent object. Due to instabilities in the laser beam and small variations in the matter of the object, the engraved mark will bear random characteristics that are very difficult to reproduce 3 . LPUF is a good instance of image-based PUFs to be employed for anti-counterfeiting purposes. Indeed, it can be engraved in several objects with various materials and it can be very small. It is also robust against aging especially when embedded on the bulk of the object.

For evaluation purpose, we manufactured samples with engraved marks of diameter $60 \mu \mathrm{m}$. Assuming a locality principle, i.e. that random variations will behave as independent events when occurring at different locations on the object, security can be improved by increasing the size of the mark or by engraving multiple marks on a physical object.

In our evaluation, we set extraction parameters providing a robustness level of $94 \%$ for a dataset of 20 different LPUFs observed 100 times each 4 . The security property $\beta$ is then evaluated using another dataset containing $R=1000$ different

\footnotetext{
${ }^{3}$ We refer to [6] for a detailed description of the process.

${ }^{4}$ For the sake of completeness, we note that the extraction parameters are set as follows: Gabor hashing parameters are fixed to $\left(\nu_{0}, F, a, \Delta\right)=(\pi / 3,4,10,30)$ and a $\mathrm{BCH}(255,131,18)$ is used for fuzzy extraction. This yields the binary output $z$ with 131 bits. For further details on the definitions of parameters, we refer to [6].
} 
LPUFs observed one time. Using the approach described in Section 7.2, the probability of collisions between outputs of different PUFs (Eq11) is estimated to be $\beta=10^{-5}$. The birthday paradox theory states that if $q$ elements are drawn from a discrete uniform distribution with range $[1, d]$, the probability of collision is $1-\left(\frac{d-1}{d}\right)^{q \cdot(q-1) / 2}$. Applying this formula with $d=1 / \beta$ allows computing the probability of collision for a given number $q$ of PUFs.

Based on the aforementioned locality principle, this means we could obtain a reasonable security level by increasing the size of the mark (or the number of marks) by a factor between 4 and 16 . Considering the $60 \mu \mathrm{m}$ diameter of the original mark, this seems a very practical option.

We also compare the obtained results with the typical results given by human biometrics. In biometrics, e.g., for fingerprints verification systems, the performance of the system is often evaluated by False Rejection Rate (FRR), False Acceptance Rate (FAR) and Equal Error Rate (EER). FRR is the probability for a valid user to be incorrectly rejected and FAR is the probability of an imposter to be incorrectly matched to the biometric of a valid user. EER is the rate at which both FAR and FRR are equal. Therefore, for the image-based physical function system, FRR and FAR can be considered to be equivalent to $1-\rho$ and $\beta$ respectively. The equivalent ERR is given when $1-\rho=\beta$ and is obtained as $E E R_{g}=0.2 \%$. In this view, our example of image-based physical function system provides better results with respect to typical fingerprints verification systems $(E E R>2 \%)$.

\section{Conclusion}

The protocol we propose is simple and reasonably efficient. It relies on a natural property that can be expected from any image-based Physical Function System, namely the fact that each object presents a unique, typical visual aspect. This is presumably easier to achieve in practice than more advanced properties such as the availability of a large amount of independent challenge-response pairs. From a computational point of view, product registration (resp. verification) requires one execution of an asymmetric cryptographic primitive, which is in line with the cost and computing capabilities expected from an image processing-capable device.

The protocol allows a simple key management policy, where critical, private keys can be kept under control of the trademark owner, whereas only public keys need to be distributed towards the (more difficult to control) verifiers. It also allows straightforward extensions, e.g. integrating a Public Key Infrastructure (PKI) for better scalability.

Experiments confirm that some Physical Function Systems, such as laser engraving, seem to bear the necessary properties to be integrated in our construction and provide a practical anti-counterfeiting system.

Eventually, our results also confirm that the security framework in [17] can be used to bridge the gap between engineering constraints and cryptographic protocols. While the previous work in [6] shows that metrics such as unclonabilibilty 
and robustness can indeed be estimated for real-world PUFs, the security analysis in this paper confirms that these metrics can also be connected to standard cryptographic analyses, using sound (and tight) reductions.

Acknowledgments. This research work was supported by the Belgian Walloon Region project TRACEA. François-Xavier Standaert is an Associate Researcher of the Belgian Fund for Scientific Research (FNRS-F.R.S.). This work has been funded in part by the ERC project 280141 (acronym CRASH).

\section{References}

1. Staake, T., Fleisch, E.: Countering Counterfeit Trade: Illicit Market Insights, BestPractice Strategies, and Management Toolbox, 1st edn. Springer Publishing Company, Incorporated (2010)

2. Bastia, S.: Next generation technologies to combat counterfeiting of electronic components. IEEE Trans. on Components and Packaging Tech. 25, 175-176 (2002)

3. Chong, C.N., et al.: Anti-counterfeiting with a random pattern. In: Int. Conf. on Emerging Security Information, Systems and Tech., pp. 146-153 (2008)

4. Pappu, R.: Physical one-way functions. PhD thesis, MIT (March 2001)

5. Pappu, R., et al.: Physical one-way functions. Science 297 (2002)

6. Shariati, S., Standaert, F.-X., Jacques, L., Macq, B.: Comprehensive study of image-based physical function system. Submitted to Journal of Cryptographic Engineering

7. Tuyls, P., Škorić, B.: Strong authentication with physical unclonable functions. In: Security, Privacy, and Trust in Modern Data Management, pp. 133-148 (2007)

8. Tuyls, P., et al.: Secure key storage and anti-counterfeiting, pp. 255-268. Springer (2008)

9. Tuyls, P., Batina, L.: RFID-Tags for Anti-counterfeiting. In: Pointcheval, D. (ed.) CT-RSA 2006. LNCS, vol. 3860, pp. 115-131. Springer, Heidelberg (2006)

10. Bulens, P., Standaert, F.-X., Quisquater, J.-J.: How to Strongly Link Data and its Medium: the Paper Case. IET Information Security 4(2), 125-136 (2010)

11. Kirovski, D.: Anti-counterfeiting: Mixing the physical and the digital world. In: Guajardo, J., Preneel, B., Sadeghi, A.-R., Tuyls, P. (eds.) Foundations for ForgeryResilient Cryptographic Hardware, vol. 09282 in Dagstuhl Seminar Proceedings (2010)

12. Buchanan, J.D.R., Cowburn, R.P., Jausovec, A.V., Petit, D., Seem, P., Xiong, G., Atkinson, D., Fenton, K., Allwood, D.A., Bryan, M.T.: Fingerprinting documents and packaging. Nature, 475 (2005)

13. Kirovski, D.: Toward an automated verification of certificates of authenticity. In: Proceedings of the 5th ACM Conference on Electronic Commerce, EC 2004, pp. 160-169. ACM (2004)

14. Chen, Y., Mihçak, K., Kirovski, D.: Certifying authenticity via fiber-infused paper. SIGecom Exch. 5, 29-37 (2005)

15. Chong, C.N., Jiang, D.: Anti-counterfeiting using phosphor puf. In: International Conference on In Anti-Counterfeiting, pp. 59-62 (2008)

16. Shariati, S., Standaert, F.-X., Jacques, L., Macq, B., Salhi, M.A., Antoine, P.: Random profiles of laser marks. In: WIC Symposium on Information Theory in the Benelux, pp. 27-34 (2010) 
17. Armknecht, F., Maes, R., Sadeghi, A.R., Standaert, F.X., Wachsmann, C.: A formalization of the security features of physical functions. In: IEEE Symposium on Security and Privacy, pp. 397-412 (2011)

18. Bauder, D.W.: An anti-counterfeiting concept for currency systems. Technical Report PTK-11990, Sandia National Labs, Albuquerque, NM (1983)

19. Commission on Engineering Committee on Next-Generation Currency Design and National Research Council Technical Systems. Counterfeit Deterrent Features for the Next-Generation Currency Design. The National Academies Press (1993)

20. Tuyls, P., Schrijen, G.-J., Škorić, B., van Geloven, J., Verhaegh, N., Wolters, R.: Read-Proof Hardware from Protective Coatings. In: Goubin, L., Matsui, M. (eds.) CHES 2006. LNCS, vol. 4249, pp. 369-383. Springer, Heidelberg (2006)

21. Gassend, B., Clarke, D., van Dijk, M., Devadas, S.: Silicon physical random functions. In: ACM Conference on Computer and Communications Security (November 2002)

22. Lim, D., Lee, J.W., Gassend, B., Suh, G.E., van Dijk, M., Devadas, S.: Extracting secret keys from integrated circuits. IEEE Transactions on Very Large Scale Integration (VLSI) Systems 13(10), 1200-1205 (2005)

23. Lee, J.W., Lim, D., Gassend, B., Suh, G.E., van Dijk, M., Devadas, S.: A technique to build a secret key in integrated circuits for identification and authentication applications. In: VLSI Circuits. Digest of Technical Papers, pp. 176-179 (2004)

24. Guajardo, J., Kumar, S.S., Schrijen, G.-J., Tuyls, P.: FPGA Intrinsic PUFs and Their Use for IP Protection. In: Paillier, P., Verbauwhede, I. (eds.) CHES 2007. LNCS, vol. 4727, pp. 63-80. Springer, Heidelberg (2007)

25. Shariati, S., Jacques, L., Standaert, F.-X., Macq, B., Salhi, M.A., Antoine, P.: Randomly driven fuzzy key extraction of uncloneable images. In: International Conference on Image Processing, ICIP (2010)

26. Tuyls, P., Skoric, B.: Secret key generation from classical physics. Philips Research Book Series (2005)

27. Škorić, B., Tuyls, P., Ophey, W.: Robust Key Extraction from Physical Uncloneable Functions. In: Ioannidis, J., Keromytis, A., Yung, M. (eds.) ACNS 2005. LNCS, vol. 3531, pp. 407-422. Springer, Heidelberg (2005)

28. Lim, D., Lee, J.W., Gassend, B., Edward Suh, G., van Dijk, M., Devadas, S.: Extracting secret keys from integrated circuits. IEEE Transactions on VLSI Systems 13(10), 1200-1205 (2005)

29. Armknecht, F., Maes, R., Sadeghi, A.R., Sunar, B., Tuyls, P.: Memory LeakageResilient Encryption Based on Physically Unclonable Functions. In: Matsui, M. (ed.) ASIACRYPT 2009. LNCS, vol. 5912, pp. 685-702. Springer, Heidelberg (2009)

30. Dodis, Y., Reyzin, L., Smith, A.: Fuzzy Extractors: How to Generate Strong Keys from Biometrics and Other Noisy Data. In: Cachin, C., Camenisch, J.L. (eds.) EUROCRYPT 2004. LNCS, vol. 3027, pp. 523-540. Springer, Heidelberg (2004)

31. Katz, J., Lindell, Y.: Introduction to Modern Cryptography. Chapman and Hall/CRC Press (2008) 